\title{
Real Time Vehicle Tracking and Automatic Engine Lock System
}

\author{
A.Arivazhagan ${ }^{1}$, R.Dhinesh ${ }^{2}$ \& R.Gomathi ${ }^{3}$ \\ ${ }^{1,2}$ UG Student, IFET College of Engineering, Gengarampalayam, Villupuram, India. \\ ${ }^{3}$ Associate Professor in ECE, IFET College of Engineering, Gengarampalayam, Villupuram, India. \\ DOI: 10.38177/ajast.2020.4303
}

The current scenario in the world is that almost all people have their own vehicle for conveyance. As, vehicle has become the most integral part of mankind. It travels as a part and parcel of man's life. Theft happens increasingly at one place or the other, when there is improper surveillance. The safety of vehicles is extremely essential in all areas, especially in public places. In this work, the vehicle tracking and locking system are done in order to satisfy the needs of security of the vehicle. Vehicle theft handling is one of the important crises in today's world. Countries which are densely populated like India finds it very difficult to track the vehicle after theft. This system consist an advanced microprocessor board which is connected to the GPS to get the real time location of the vehicle which is then connected to the mobile application through IoT platform. Mobile application will show the location of the vehicle in a map and also the places surrounding the vehicle by capturing it through camera connected to the system. Not only to identify the theft but also it avoids the theft by having dual switching method both on vehicle and the mobile application connected to the vehicle via Bluetooth. It also gives the alert notification in the mobile if someone takes the vehicle when it is turned on in the mobile.

Keywords: Mobile START/STOP app, GPS, Camera, Raspberry Pi, Motor \& Motor Driver, Mobile Tacking \& Image app.

\section{Introduction}

The globe is running into a tireless and a comfortable manner for all individuals to compute from one place to another place by various modes of transport. One such thing is the transportation through road which is very easy for mankind. It easily makes a conveyance through two or four wheelers. These vehicles play a very vital role in man's life. Such usage of vehicles is necessary and it is more than important to safeguard these vehicles in all places. The safety and security of the vehicles is essential so that people do not suffer after the theft. There are many systems that prevail in order to make the journey of people in a good and comfortable manner. The system proposed mainly revolves around real time vehicle tracking, theft detection and engine locking system.

Vehicle tracking is very essential in almost every place a vehicle is parked in regard to safety. Inspite of surveillance there will be certain flaws that would occur is none other than theft. This should not be violated at any cost and that should be curtailed then and there. In order to have a continuous check or monitoring a lot of systems have been proposed for the safe and secure conditions of the vehicles, especially two and four wheelers.

This work has a stream of process starting from the start/stop command to start or stop the vehicle in the mobile start/stop app till the vehicle tracking and image through the mobile tracking and image app with the help of raspberry pi, camera GPS. It helps in tracking and easy identification of the vehicle.

\section{Literature Survey}

June Myint Mo Khin, Dr. Nyein Nyein O [1] have proposed a system in which the Arduino \& GSM controls the system. The place is identified by the GPS and the information received is stored in the database. Ruipeng Gao, Mingmin Zhao, Tao Ye, Fan Ye, Yizhou Wang, Guojie Luo [2] have discussed a method that tracks and records the vehicle parking location with the help of inertial data and all the sensing and computing will process locally in phone. Saima Safdar, Anwar Zeb, Ajmal Khan and Zeeshan Kaleem [3] have delivered a method in which the GPS 
locates the system with the latitude and longitude, with the GUI the vehicles can be tracked easily. Sathe Pooja [4] has proposed a method that would help to trace the location of the vehicle with the help of the GPS through an SMS. Shihab A. Hameed, Othman Khalifa, et, el [5] have proposed a method in which the car can be tracked easily through GPS and an alert notification will be sent to the user's mobile and then the vehicle can be retrieved. $\mathrm{H}$. Song, S. Zhu, and G. Cao [6] have delivered a method in which based on the sensors the vehicle that is lost can be retrieved. Tahesin Attar, Prajakta Chavan, Vidhi Patel, Megha Gupta, Debajyoti Mukhopadhyay [7] have discussed a method on the basis of IoT that has safety for the user, keyless locking and unlocking type and has reliability for a car alone. Zhigang Liu, Anqi Zhang and Shaojun Li [9] have discussed a system that is mainly controlled by RFID and then with the help of the IoT the location is identified by the user.

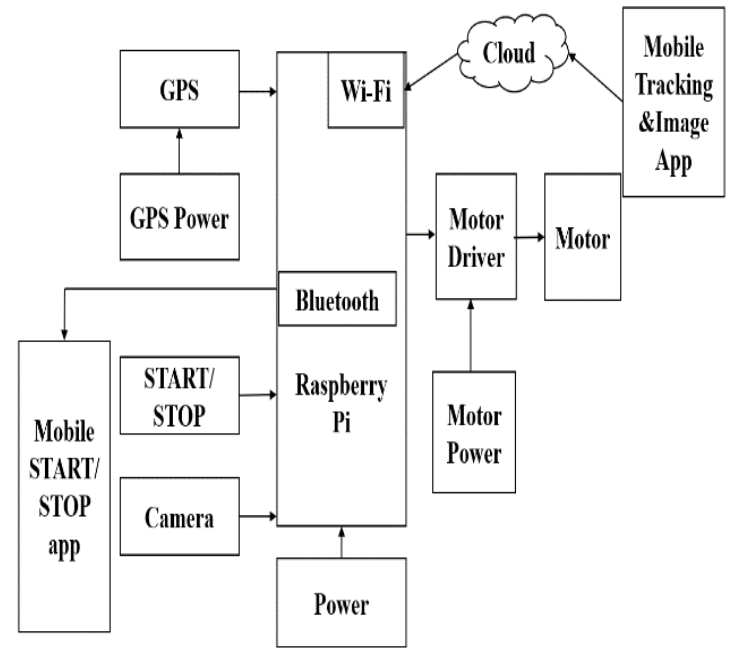

FIG 1: Block Diagram

\section{Existing System}

The prevailing system is based on the extraction of vehicle parts with pre-trained appearances and geometric models to avoid drift to irrelevant objects. In addition to this, well-structured on-road environment to generate a road model with better observations and efficient predictions is present. But the systems that exist has few flaws such as no safety for the user life, high computational cost, surveillance is improper, theft cannot be identified, long distance communication is not possible. All the above-mentioned flaws can be overcome by the proposed system.

\section{Proposed System}

The idea centred in this system is to design and develop a vehicle tracking and locking system in the real time environment in order to ensure safety of the vehicle whenever or wherever the vehicle is parked. The vehicles that are often used for easy conveyance by human beings are mostly two and four wheelers. This paper will clearly give a safe, secure and a very helpful methodology for both two and four wheelers.

The system uses Raspberry Pi as the main unit of the entire system. It uses two mobile applications in order to have a control and track of the vehicle at unsecure moments and can easily identify the vehicle and acquire it back. The two mobile applications are mobile start/stop app by MIT APP INVENTOR and the other one is mobile tracking and image application by Blynk app.

GPS is present which helps in locating the position of the vehicle, with latitude and longitudinal positions of the vehicle. There is a presence of a camera that aids in capturing the image of the vehicle and sends the data with the help of other components. Here, instead of the vehicle a motor and a motor driver are connected as a simulation for the vehicle.

\section{Working}

The system's working, starts from the mobile start/stop app. This is an app developed for the START/STOP format. By this device the user of the vehicle can click on to start or stop, if he wants to ride or not to ride the vehicle 
respectively. Only by this command the vehicle will be enabled to move and not by any other means. The mobile start/stop app and the raspberry pi are connected with the Bluetooth. From the mobile start/ stop app the command is sent to the Bluetooth and that in turn sends it to the raspberry pi and that responds to the commands START, if the vehicle needs to be driven and STOP, it does not move according to the user's command. Instead of the vehicle, the motor and the motor driver are present as a simulation of the vehicle.

The motor is driven by the motor driver. There is a presence of the mobile tracking and image app by the Blynk. This app does two functions. One function is that, with the help of GPS the current location of the vehicle is obtained with the latitude and the longitude. The other function is that, there is a presence of a camera which captures the image of the environment where the vehicle is present.

If in case at unavoidable conditions the parked vehicle is moved without the user's command through the app, the notification will be sent to the mobile tracking and image app with location of the vehicle and the location of the vehicle, the owner can lock the engine by giving the stop command and easily identify the place and acquire the vehicle.

Thus, this paves for an excellent surveillance method for the vehicle, a good tracking system and an efficient engine locking system.

\section{Mobile Start/Stop App}

This app has been developed in order to enable the vehicle's movement based on the user of the vehicle in this system. The creation of this app is made easy because of the blocks present in it.

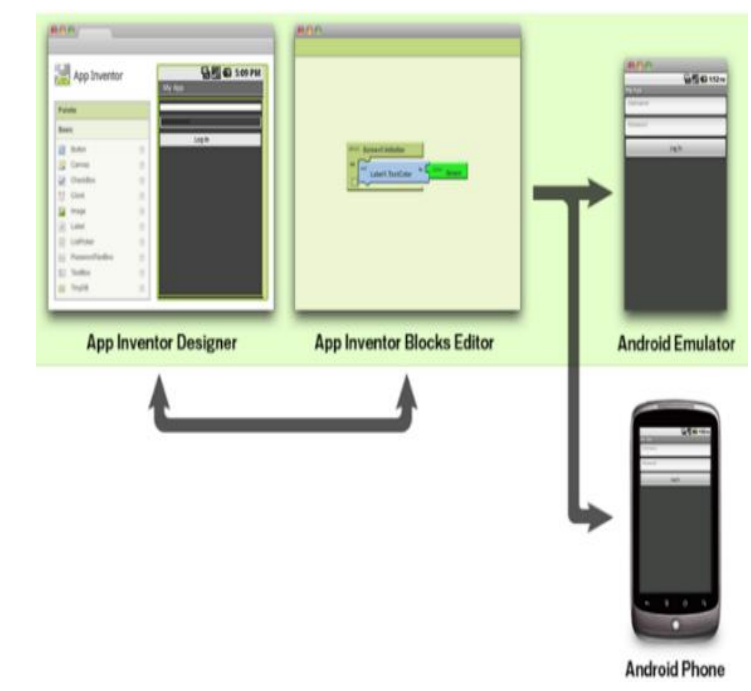

FIG 2: Mobile START/STOP App-MIT APP INVENTOR

\section{Mobile Tracking \& Image App}

This is done by Bkynk, a platform in which it is capable of performing few functions. It performs two functions here in this system. One function with the help of GPS the current location of the vehicle is obtained with the latitude and the longitude. The other function is with the presence of a camera which captures the image of the environment where the vehicle is present. At unavoidable conditions the parked vehicle is moved without the user's 
command through the app, the notification will be sent to the mobile tracking and image app with location of the vehicle and the location of the vehicle. The owner can lock the engine by giving the stop command and easily identify the place and acquire the vehicle.

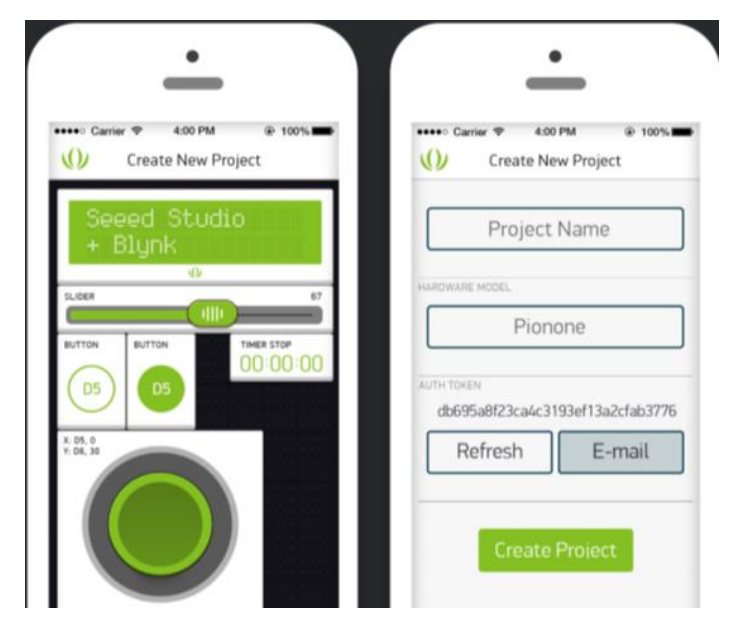

FIG 3: Blynk Branded Interface

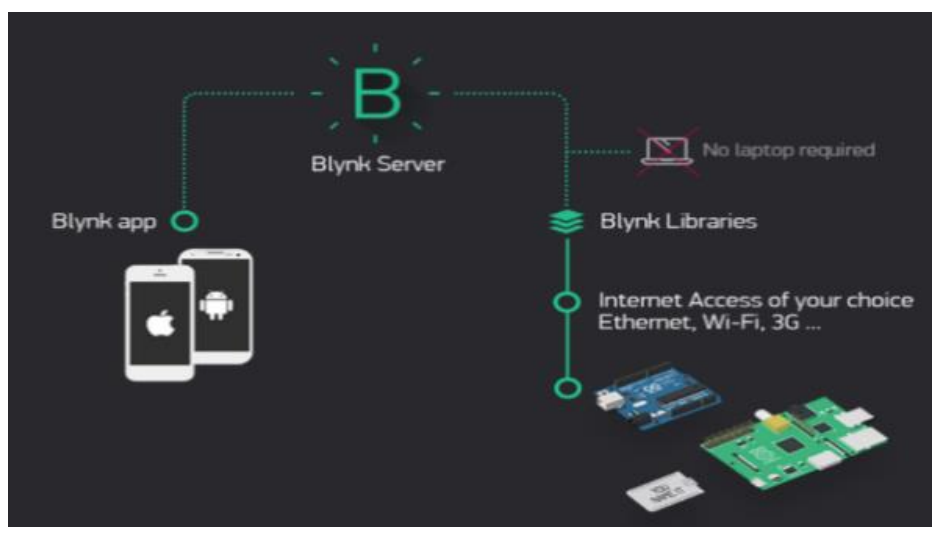

FIG 4: Blynk Infrastructure

\section{Tools Used}

\section{Start/Stop Switch}

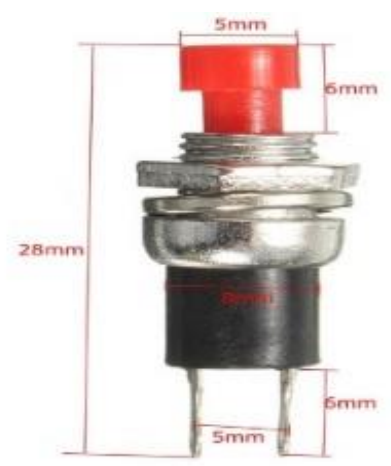

FIG 5: START/STOP Switch

This is a tool that is used for starting and stopping the vehicle, which is connected in regard to the mobile start and stop app. It will work with respect to the command given by the user. 


\section{Camera}

This is QHM 495LM camera used to capture the image of the environment that is surrounded at vehicle's location.

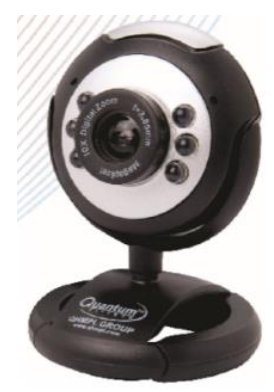

FIG 6: Camera

\section{Motor and Motor Driver}

Motor device is used instead of the vehicle. It is used as the simulation for the vehicle. BO (Battery operated) Series 1 100RPM DC Motor (Straight) device is used. The motor is driven by the motor driver (L293D).

\section{Raspberry Pi}

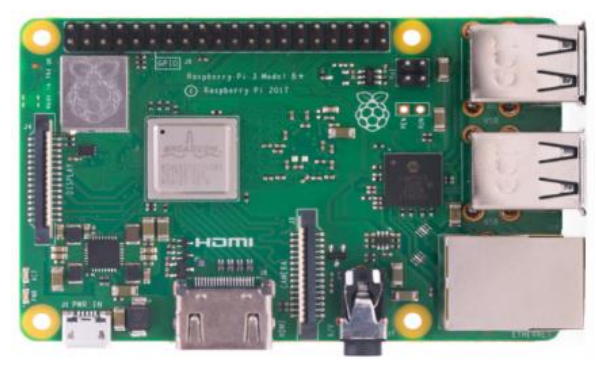

FIG 7: Raspberry Pi

Here, Raspberry Pi 3 Model B+ is used as brain of the entire system. It connects to all the devices in the method and gives the desired output.

\section{Global Positioning System}

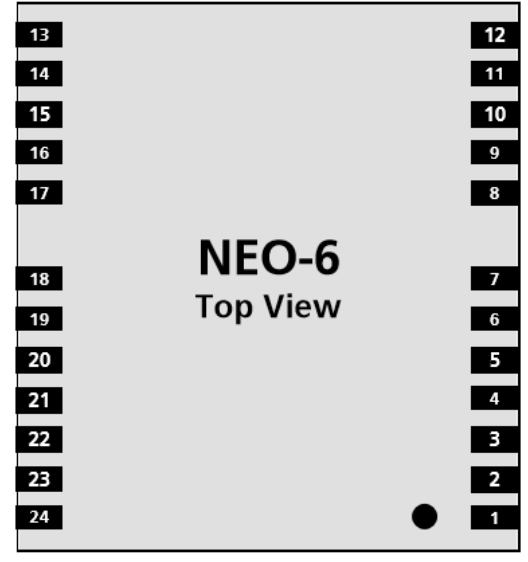

FIG 8: Global Positioning System 
Asian Journal of Applied Science and Technology

Volume 4, Issue 3, Pages 17-22, July-September 2020

The Technical data sheet NEO-6 series of GPS modules is used to locate the position of the vehicle in latitude and longitude. It helps the user to identify the correct location of their vehicle.

\section{Conclusion}

The Real Time Vehicle Tracking System and Automatic Engine Lock System is exclusively used for vehicle tracking, theft detection and engine locking system. It formulates various security measures for the vehicle. The system provides a stream of process starting from the start/stop of the engine till the tracking and image of the vehicle along with the surrounded environment. The data is obtained by a stream of devices starting from the mobile start/stop app for the user to decide, then the location of the vehicle with the help of GPS. When the vehicle is parked and there is an attempt of theft it alerts the owner through a notification along with the location and image of the vehicle with help of cloud and the mobile tracking and image app. The system is cost effective, very efficient, free from theft and secure. The future enhancement of this system will be made compact, integration of GSM will be made to get continuous data of the system, and robust.

\section{References}

[1] June Myint Mo Khin, Dr. Nyein Nyein O, "Real-Time Vehicle Tracking System Using Arduino, GPS, GSM and Web-Based Technologies", International Journal of Science and Engineering Applications Volume 7-Issue 11,433-436, 2018, ISSN:-2319-7560.

[2] Ruipeng Gao, Mingmin Zhao, Tao Ye, Fan Ye, Yizhou Wang, Guojie Luo, "Smartphone-based Real Time Vehicle Tracking in Indoor Parking Structures”, 1536-1233.

[3] Saima Safdar, Anwar Zeb, Ajmal Khan and Zeeshan Kaleem “Android based vehicle tracking system”, EAI Endorsed Transactions on Energy Web and Information Technology 012018 - 042018 | Volume 5 | Issue 17 | e5.

[4] Sathe Pooja, "Vehicle Tracking System Using GPS", International Journal of Science and Research (IJSR), India Online ISSN: 2319-7064 Volume 2 Issue 9, September 2013.

[5] Shihab A. Hameed, Othman Khalifa, et, el, "Car Monitoring, Alerting and Tracking Model Enhancement with Mobility and Database Facilities," International Conference on Computer and Communication Engineering (ICCCE 2010), pp.1-5, May 2010.

[6] H. Song, S. Zhu, and G. Cao, "Svats: A sensor-network-based vehicle anti-theft system," IEEE INFOCOM 2008, pp.2128-2136, April.2008.

[7] Tahesin Attar, Prajakta Chavan, Vidhi Patel, Megha Gupta, Debajyoti Mukhopadhyay, “An Attempt to Develop an IOT based Vehicle Security System", 0-7695-6618-9/18.

[8] Yongkun Fang, Chao Wang, Wen Yao, Xijun Zhao, Huijing Zhao and Hongbin Zha, "On-Road Vehicle Tracking Using Part-Based Particle Filter”, 1524-9050 @ 2019 IEEE.

[9] Zhigang Liu, Anqi Zhang and Shaojun Li, "Vehicle Anti-Theft Tracking System Based on Internet of Things", Internationa Conference on Computer and Communication Engineerin (ICCCE 2010), pp.15, May.2010. 